\title{
Association of Inflammatory and Oxidative Stress Markers with Metabolic Syndrome in Asian Indians in India
}

\author{
Veena S. Rao, ${ }^{1}$ Radhika K. Nagaraj, ${ }^{2}$ Sridhara Hebbagodi, ${ }^{2}$ \\ Natesha B. Kadarinarasimhiah, ${ }^{1}$ and Vijay V. Kakkar ${ }^{1,2}$ \\ ${ }^{1}$ Tata Proteomics and Coagulation Unit, Thrombosis Research Institute, Narayana Hrudayalaya, 258/A Bommasandra Industrial Area, \\ Anekal Taluk, Bangalore 560099, India \\ ${ }^{2}$ Emmanuel Kaye Bioinformatics Centre, Thrombosis Research Institute, Bangalore 560099, India
}

Correspondence should be addressed to Veena S. Rao, veenasrao@triindia.org.in

Received 30 September 2010; Revised 29 October 2010; Accepted 5 November 2010

Academic Editor: Rei Shibata

Copyright (C) 2011 Veena S. Rao et al. This is an open access article distributed under the Creative Commons Attribution License, which permits unrestricted use, distribution, and reproduction in any medium, provided the original work is properly cited.

\begin{abstract}
Metabolic syndrome (MetS) is a primary risk factor for cardiovascular disease and is associated with a proinflammatory state. Here, we assessed the contribution of inflammatory and oxidative stress markers towards prediction of MetS. A total of 2316 individuals were recruited in Phase I of the Indian Atherosclerosis Research Study (IARS). Modified ATPIII guidelines were used for classification of subjects with MetS. Among the inflammatory and oxidative stress markers studied, levels of hsCRP $(P<.0001)$, Neopterin $(P=.036)$, and oxLDL $(P<.0001)$ were significantly higher among subjects with MetS. Among the markers we tested, oxLDL stood out as a robust predictor of MetS in the IARS population (OR 4.956 95\% CI 2.504-9.810; $P<.0001$ ) followed by hsCRP (OR 1.324 95\% CI 1.070-1.638; $P=.010$ ). In conclusion, oxLDL is a candidate predictor for MetS in the Asian Indian population.
\end{abstract}

\section{Introduction}

Metabolic syndrome (MetS) is a constellation of metabolic abnormalities that confer increased risk of cardiovascular disease (CVD) and diabetes mellitus (DM) [1]. The criteria for the metabolic syndrome have evolved since the original definition by the World Health Organization in 1998 [2], reflecting growing clinical evidence and analysis by a variety of consensus conferences and professional organizations $[3,4]$. The major features of MetS include central obesity, hypertriglyceridemia, low HDL cholesterol, hyperglycemia, and hypertension.

The accepted and unifying hypothesis to describe the pathophysiology of metabolic syndrome is insulin resistance, caused by a defect in insulin action that is not fully understood. Free fatty acids (FFAs) are released in abundance from an expanded atherogenic adipose tissue mass leading to hyperinsulinemia [5]. Recent evidence indicates that a proinflammatory state is superimposed and contributes to the insulin resistance produced by excessive FFAs $[6,7]$. Adipose tissue-derived macrophages may be the primary source of proinflammatory cytokines [8], and it has been reported that enhanced secretion of interleukin 6 (IL-6) and tumor necrosis factor (TNF- $\alpha$ ) by adipocytes and monocytederived macrophages leads to more insulin resistance and lipolysis of adipose tissue triglyceride stores increasing circulating levels of FFAs [9]. IL-6 and other cytokines also enhance hepatic glucose production, VLDL production by the liver, and insulin resistance in the muscle. Cytokines and FFAs are also known to increase hepatic production of fibrinogen and adipocyte production of plasminogen activator inhibitor 1 (PAI-1) resulting in a prothrombotic state [5]. Higher levels of circulating cytokines stimulate the hepatic production of C-reactive protein (CRP) [10] and reduced production of the anti-inflammatory and insulin sensitizing cytokine, adiponectin has been associated with MetS [11]. Studies using cellular and animal models have described the role of oxidized LDL in the pathophysiology of incident MetS [12,13]. This hypothesis is supported by population-based prospective human studies. The CARDIA study found increased incidence of MetS in individuals with higher circulating levels of ox-LDL with accumulation of 
three of its risk factor constituents: obesity, hyperglycemia and hypertriglyceridemia [14]. Both oxidative stress and inflammatory stress associated with MetS have been found to initiate and propagate atherosclerotic macrovascular disease [15].

The multitude of risk factors amalgamating into MetS is increasing in Indians to reach epidemic proportions, which may present the common ground that enhances CVD risk in this ethnic group. The peculiar dyslipidemic obesity phenotype of Asian Indians with higher truncal and abdominal fat at lean body mass predisposes this ethnic group to the consequences of a proinflammatory and prothrombotic state [16]. Thus it is appealing to pursue a comprehensive CAD risk assessment with an understanding of the association of inflammation between MetS. Here, we assessed the association of inflammatory biomarkers with MetS and their independent ability to predict MetS in the studied population.

\section{Materials and Methods}

The Indian Atherosclerosis Research Study (IARS) is an ongoing, family-based epidemiological study investigating the genetic, conventional and environmental factors associated with $\mathrm{CAD}$ in Asian Indians living in the Indian subcontinent. Novel biomarker discovery is a specific aim of this study. For the study, families were enrolled from two Indian cities: Bangalore and Mumbai. Subjects were recruited through a proband who showed: (i) angiographic evidence of CAD (males $\leq 60$ years and females $\leq 65$ years at onset), (ii) a family history of CAD/CVD and, (iii) underwent therapeutic/surgical treatment at participating hospitals. Extended family members (both affected and unaffected) were enrolled, provided they met the recruitment age of 18 or above. Individuals with a history of any other major illness or concomitant infections were excluded from the study. Information pertaining to demographics; life style; anthropometrics; medical history of diabetes, hypertension and CAD; medication details, and a threegeneration pedigree were recorded for each participant. Fasting sugar levels of $>126 \mathrm{mg} / \mathrm{dl}$ were considered diabetic and, a systolic/diastolic blood pressure of $>140 / 90 \mathrm{~mm} \mathrm{Hg}$ was considered hypertensive. The study was conducted following the guidelines defined by the Indian Council of Medical Research and the Declaration of Helsinki for undertaking human clinical research. An institutional ethics committee approved the IARS and voluntary, signed informed consent was obtained from all participants.

Blood was collected by venipuncture after an overnight fast and processed using standardized protocols at each collection site. Blood was centrifuged for $10 \mathrm{~min}$ at $4{ }^{\circ} \mathrm{C}$, aliquoted in $0.5-1 \mathrm{~mL}$ volumes of sodium-citrate plasma, EDTA plasma and serum, and stored at $-80^{\circ} \mathrm{C}$ within $2 \mathrm{~h}$ of venipuncture. For protein measurements, samples were thawed on ice and aliquoted into bar-coded Eppendorf tubes. The new sample aliquots were refrozen to $-80^{\circ} \mathrm{C}$ until time of testing at which point they were thawed on ice again. Thus, samples from each collection site were exposed to identical numbers of freeze-thaw cycles for a given assay ensuring that sample handling does not contribute to any significant differences in protein levels.

2.1. Conventional Risk Factors. Serum total cholesterol and triglyceride were estimated by standard enzymatic analysis using reagents, standards and controls from Randox Laboratories Ltd. (Antrim, UK). The concentration of high-density lipoprotein (HDL)-cholesterol was estimated after precipitation of non-HDL fractions with a mixture of $2.4 \mathrm{mmol} / \mathrm{L}$ phosphotungstic acid and $39 \mathrm{mmol} / \mathrm{L}$ magnesium chloride. The concentration of low-density lipoprotein (LDL)cholesterol was calculated using the Fridewald formula.

2.2. Measurement of Candidate Biomarkers. The candidate protein markers of vascular disease were measured in plasma (EDTA or citrate) or serum using solid-phase immunoassays and immunoturbidometric assays with commercially available reagents. Plasma interleukin (IL) 6 level and adiponectin was measured by enzyme-linked immunosorbent assa, (ELISA) from R \& D Systems (Minneapolis, USA) and plasma hsCRP level was measured using the Roche latex Tina quant kit (Roche Diagnsotics, Basel, Switzerland). Levels of secretory phospholipase A2 (sPLA2) were determined using a sandwich immunometric assay (Cayman Corporation, Michigan, USA); myeloperoxidase using the sandwich enzyme immunoassay using kits from Mercodia (Uppasala, Sweden), neopterin using the enzyme immunoassay kit from IBL (Hamburg, Germany), oxLDL using the commercially available sandwich ELISA kit from Mercodia (Uppasala Sweden) and Leptin using the ELISA kit from Bio-Line (Bruxelles, Belgium). In the oxLDL ELISA, murine monoclonal antibody, mAb-4E6 was used as capture antibody and the second antibody was a peroxidaseconjugated anti-apolipoprotein $\mathrm{B}$ antibody recognizing oxLDL bound to the solid phase.

2.3. Quality Control. Our quality control program included evaluation of intra-assay variations between duplicate sample measurements and inter-assay variations between independent repeat experiments. Sample measurements with coefficient of variation, CV $>15 \%$ were either retested or excluded from the dataset. The inter-assay CV for the commercial controls and NHP ranged from $4.9 \%$ to $7.0 \%$ for total cholesterol, $6.1 \%$ to $7.7 \%$ for triglyceride, $7.1 \%$ to $12.2 \%$ for HDL-cholesterol, $3.3 \%$ to $5.2 \%$. The inter-assay CV for IL-6 relative to NHP was 4.3\%; for plasma hsCRP 7.85\%; secretory phospholipase A2 (sPLA2) was 5.37\%; the inter-assay CV for NHP and the low and high controls provided by the manufacturer along with the kit ranged from 5.99 to $11.8 \%$ for neopterin and from 4.4 to $16 \%$ for oxLDL.

2.4. Statistical Analysis. SPSS ver. 17.0 statistical packages were used in the analysis. A $P$-value $<.05$ was considered statistically significant. The modified NCEP-ATPIII procedure was used to classify the IARS cohort into those with/without MetS based (Table 1). A cross-classification of MetS groups and CAD status was obtained. 
TABLe 1: Metabolic syndrome: diagnostic criteria.

\begin{tabular}{|c|c|}
\hline Modified ATPIII criteria & WHO criteria \\
\hline $\begin{array}{l}\text { (i) Abdominal density as } \\
\text { defined by waist circumference } \\
\text { cuts off } \geq 90 \mathrm{~cm} \text { for men and }\end{array}$ & $\begin{array}{l}\text { (i) Fasting blood glucose level } \\
\text { of } \geq 110 \mathrm{md} / \mathrm{dl}(6.1 \mathrm{mmol} / \mathrm{L}) \\
\text { or Diabetic }\end{array}$ \\
\hline$\geq 80 \mathrm{~cm}$ for women & (ii) WHR of $\geq 0.89$ in men, \\
\hline (ii) BMI cuts off at $>23 \mathrm{~kg} / \mathrm{m}^{2}$ & $\begin{array}{l}>0.81 \text { in women and BMI }> \\
23 \mathrm{~kg} / \mathrm{m}^{2}\end{array}$ \\
\hline $\begin{array}{l}\text { (iii) Serum TG } \geq 150 \mathrm{mg} / \mathrm{dl} \\
(1.7 \mathrm{mmol} / \mathrm{L})\end{array}$ & $\begin{array}{l}\text { (iii) Hypertriglyceridemia } \\
\text { with TG }>150 \mathrm{mg} / \mathrm{dl}\end{array}$ \\
\hline $\begin{array}{l}\text { (iv) } \mathrm{HDL}-\mathrm{C}<40 \mathrm{mg} / \mathrm{dl} \text { in men } \\
(1.03 \mathrm{mmol} / \mathrm{L}) \text { and }<50 \mathrm{mg} / \mathrm{dl} \\
(1.29 \mathrm{mmol} / \mathrm{L}) \text { in women }\end{array}$ & $\begin{array}{l}\text { (iv) HDL-C }<35 \mathrm{mg} / \mathrm{dl} \text { in men } \\
\text { and }<39 \mathrm{mg} / \mathrm{dl} \text { in women }\end{array}$ \\
\hline (v) Blood pressure $\geq 130 / 85$ & $\begin{array}{l}\text { (v) High Blood Pressure } \\
>140 / 90 \mathrm{~mm} \mathrm{Hg} \text { or }\end{array}$ \\
\hline $\begin{array}{l}\text { (vi) Fasting blood glucose level } \\
\text { of } \geq 110 \mathrm{md} / \mathrm{dl}(6.1 \mathrm{mmol} / \mathrm{L})\end{array}$ & $\begin{array}{l}\text { documented evidence of } \\
\text { anti-hypertensive therapy }\end{array}$ \\
\hline
\end{tabular}

Logarithmic transformation was performed to normalize skewed variables. Both continuous and categorical forms of the distribution of these variables were used in the analysis. The retransformed mean and standard error of the mean (SEM) are shown in tables. Differences between continuous variables were assessed using Student's $t$-test and those between categorical variables using the Chi-square test. Analysis of covariance was used to test for significance after adjusting for potential confounding variables. Odds ratios (ORs) and corresponding 95\% confidence intervals (CIs) were calculated using conditional logistic regression analysis.

The Sensitivity, Specificity and Area under ROC were calculated for each model to determine its discriminative capacity. This method tests the difference between two models in their estimated probability to correctly classify "case subject" as "case subject", and "control subject" in to a control group. This reveals the prediction ability of the model. To assess the predictive value of the biomarkers, all variables were entered into a conditional logistic regression model (taking into account the gender and age matching), and cumulative AUC was calculated using the predictive probability values.

Multinomial Logistic Regression was used to identify the contribution of the selected biomarkers using odds ratio and associated confidence interval. The groups 1 and 2 are: no MetS, and MetS with no CAD and groups 3 and 4 are MetS groups within CAD group. Multinomial Logistic Regression is similar to binary logistic regression with no restriction on the number of dependent variables.

To identify biomarkers that discriminate between MetS and no MetS groups, and to identify the threshold levels of these biomarkers for correct classification, a two-group discriminate analysis was used. The linear discriminate function is an objective statistical method used to obtain predictive scores based on two or more variables from group discrimination. The discriminate score is based on the overall mean values of each of the variables. Individuals were classified into one group or the other depending on whether the score of an individual is lesser or greater than the predictive score.

\section{Results}

3.1. Clinical and Demographic Characteristics of IARS Participants. The 2005 modified National Cholesterol Education Programme Adult Treatment Panel III (NCEP ATP III) criteria [3] were used to define MetS in the study population (Table 1). A total of 2316 individuals were categorized into two groups of subjects with or without MetS, based on the presence of a minimum of 3 out of 5 of the defining risk factors (Table 1). As expected, subjects with MetS had significantly higher systolic blood pressure (BP), diastolic BP, body mass index (BMI), circulating levels of total cholesterol, triglycerides and LDL cholesterol than those without MetS. Similarly, HDL levels were significantly lower in MetS subjects with significantly more diabetics \& hypertensive. There was no difference in the number of smokers in the two groups canceling the oxidative and inflammatory stress due to smoking on either group of subjects (Table 2). As anticipated subjects with MetS had significantly higher levels of leptin and decreased levels of adiponectin (Table 2).

Novel biomarkers for inflammatory and oxidative stress were assayed. Individuals with MetS showed most significant increases in ox-LDL $(P<.0001)$ \& hsCRP $(P<.0001)$, although sPLA2 $(P=.028)$, IL-6 $(P=.039)$ and neopterin $(P=.036)$ levels were also significantly increased (Table 3$)$.

3.2. Prediction of MetS Incidence Using Markers of Inflammation and Oxidative Stress. We used multinomial logistic regression analysis to identify biomarkers that classify subjects with risk of developing MetS. The study population was divided into 4 groups as follows: group 1-subjects without MetS (as defined by the modified ATP-III guidelines) and CAD; group 2-subjects with MetS and no CAD, group 3subjects without MetS and CAD affected, and group 4subjects with MetS and CAD affected. This study design could indicate biomarkers with a specific role in MetS by comparing groups 1 and 2 while CAD-specific markers would be obtained by comparing groups 1 and 3 . Of the four inflammatory markers studied, only hsCRP identified subjects with risk of developing MetS (OR 1.492 95\% CI 1.144-1.947; $P=.003$ ) (Table 4(a)). Among the oxidative stress markers, oxLDL best predicted MetS in our population (OR 6.031 95\% CI 2.856-12.740; $P<.0001$ ). IL-6 emerged as a CAD-specific marker and neopterin seemed to be a marker for subjects with both MetS and CAD (Table 4(b)).

Having identified the key markers, we performed logistic regression analysis to assess the interaction between these markers. Using both forward and backward logistic regression analysis, we found that all the three markers qualified for the model indicating that they independently predict risk of developing MetS. The odds ratio for development of MetS was highest for oxLDL (OR 4.956 95\% CI 2.504-9.810; $P<.0001$ ) compared to hsCRP (OR $1.32495 \%$ CI 1.070 $1.638 ; P=.010)$. In combination with oxLDL and hsCRP the odds of prediction of developing MetS was not significant for 
TABLE 2: Clinical and demographic characteristics of the study subjects.

\begin{tabular}{|c|c|c|c|}
\hline & $\begin{array}{l}\text { Individuals with MetS } \\
\qquad(n=968)\end{array}$ & $\begin{array}{l}\text { Individuals without MetS } \\
\qquad(n=1348)\end{array}$ & $P$-Value \\
\hline CAD individuals $(n)(\%)$ & $388(40.1 \%)$ & $385(28.6 \%)$ & $<.0001$ \\
\hline \multicolumn{4}{|c|}{ Continuous variables-Mean \pm SEM (retransformed mean) } \\
\hline Age & $51.689 \pm 1.102$ & $48.309 \pm 1.209$ & .864 \\
\hline $\mathrm{BMI}\left(\mathrm{kg} / \mathrm{M}^{2}\right)$ & $26.855 \pm 0.383$ & $25.036 \pm 0.420$ & $<.0001$ \\
\hline Waist hip ratio & $0.937 \pm 0.006$ & $0.918 \pm 0.007$ & .372 \\
\hline Systolic blood pressure & $129.326 \pm 1.305$ & $121.296 \pm 1.432$ & $<.0001$ \\
\hline Diastolic blood pressure & $84.838 \pm 0.760$ & $79.184 \pm 0.834$ & $<.0001$ \\
\hline Total cholesterol mg/dL* & $5.148 \pm 0.021(172.0869)$ & $5.039 \pm 0.023(154.3156)$ & .004 \\
\hline Triglycerides mg/dL* & $5.171 \pm 0.036(176.0908)$ & $4.708 \pm 0.039(110.830)$ & $<.0001$ \\
\hline HDL-Cholesterol mg/dL* & $3.591 \pm 0.020(36.2703)$ & $3.762 \pm 0.022(43.0344)$ & $<.0001$ \\
\hline LDL-Cholesterol mg/dL* & $4.550 \pm 0.034(94.6324)$ & $4.417 \pm 0.037(82.8473)$ & .051 \\
\hline Adiponectin ng/ml $(n=766)$ & $8.308 \pm 0.034(4056.1924)$ & $8.542 \pm 0.038(5125.5852)$ & .000 \\
\hline Leptin $\mathrm{ng} / \mathrm{ml}(n=543)$ & $2.888 \pm 0.070(17.9573)$ & $2.490 \pm 0.090(12.0612)$ & .001 \\
\hline \multicolumn{4}{|c|}{ Categorical Variables: $n(\%)$} \\
\hline Male & $552(57.0)$ & $802(59.5)$ & \multirow{2}{*}{.226} \\
\hline Female & $416(43.0)$ & $545(40.5)$ & \\
\hline \multicolumn{4}{|l|}{ Smoking } \\
\hline Never & $745(77.3)$ & $1076(80.1)$ & \multirow{4}{*}{.318} \\
\hline Ex-Smoker & $138(14.3)$ & $164(12.2)$ & \\
\hline Occasional & $11(1.1)$ & $19(1.4)$ & \\
\hline Current Smoker & $70(7.3)$ & $85(6.3)$ & \\
\hline Hypertension & $383(39.6)$ & $301(22.4)$ & $<.0001$ \\
\hline Diabetes & $313(32.4)$ & $196(14.6)$ & $<.0001$ \\
\hline Statin & $279(29.3)$ & $294(22.0)$ & $<.0001$ \\
\hline
\end{tabular}

${ }^{*}$ Log transformed variables represented as Mean \pm Standard error (retransformed mean). $P$-value for categorical values was obtained using chi-square test and for continuous variables using for 2 -sample $t$-test.

TABLE 3: Comparison of plasma levels of inflammatory and oxidative stress markers among subjects with and without metabolic syndrome.

\begin{tabular}{lccc}
\hline Variable & Individuals with MetS & Mean \pm SEM (retransformed mean) & $P$-value \\
\hline sPLA2 $(\mathrm{pg} / \mathrm{ml})$ & $8.119 \pm 0.046(3357.6614)$ & $7.963 \pm 0.054(2872.6780)$ & $.028^{*}$ \\
Interleukin $6(\mathrm{pg} / \mathrm{ml})$ & $1.151 \pm 0.064(3.1613)$ & $0.945 \pm 0.076(2.5728)$ & $.039^{*}$ \\
Myeloperoxidase $(\mu \mathrm{g} / \mathrm{L})$ & $5.591 \pm 0.042(268.0034)$ & $5.614 \pm 0.049(274.2390)$ & .723 \\
Neopterin $(\mathrm{nmol} / \mathrm{L})$ & $2.142 \pm 0.038(8.5164)$ & $2.020 \pm 0.044(7.5383)$ & $.036^{*}$ \\
Fibrinogen $(\mathrm{g} / \mathrm{L})$ & $1.468 \pm 0.019(4.3405)$ & $1.431 \pm 0.023(4.1828)$ & .216 \\
oxLDL $(\mathrm{mU} / \mathrm{L})$ & $10.971 \pm 0.027(58162.72706)$ & $0.515 \pm 0.107(1.6736)$ & $<.0001^{* *}$ \\
hsCRP $(\mu \mathrm{g} / \mathrm{ml})$ & $1.024 \pm 0.091(2.7843)$ & & $<.0001^{* *}$ \\
\hline
\end{tabular}

neopterin $(P=.180)$ (Figure 1$)$. ROC curve analysis using the predictive probability for the above model evaluated the utility of assaying oxLDL, hsCRP and neopterin for risk prediction. When all 3 markers were used in combination, the AUC of the model was $0.71(P<.0001)$ indicating that $71 \%$ of the study population could be classified correctly based on these markers (Figure 2).

3.3. Discriminant Analysis. To assess the percent contribution of the inflammatory and oxidative stress biomarkers to identify individuals with MetS we conducted linear discriminant analysis. As seen in Table 5(a), among all biomarkers studied, the structure correlation for ox-LDL is the highest (0.746) with a max contribution $(37.99 \%)$ to a diagnosis of MetS. Based on the Discriminant scores obtained, we could classify $63.4 \%$ of the subjects correctly.

We obtained threshold levels for these biomarkers for the detection of MetS based on the Discriminant scores (Table 5(b)). Subjects with higher than the threshold value for ox-LDL have a 5-fold risk of developing MetS. Table 5(c) depicts the mean levels of these biomarkers obtained for the four groups based on the predictive classification. 
TABLE 4

(a) Multinominal regression analysis: Odds ratio and 95\% CI for inflammatory and oxidative stress markers for subjects with and without MetS

\begin{tabular}{lcc}
\hline & OR $(95 \% \mathrm{CI})$ & $P$-value \\
\hline Inflammatory markers & & .874 \\
$\quad$ sPLA2 & $0.967(0.64-1.455)$ & .120 \\
Interleukin 6 & $1.298(0.934-1.804)$ & $.003^{* *}$ \\
hsCRP & $1.492(1.144-1.947)$ & .266 \\
$\quad$ Fibrinogen & $0.525(0.168-1.634)$ & .304 \\
Oxidative stress markers & & $<.0001^{* *}$ \\
$\quad$ Myeloperoxidase & $1.262(0.810-1.969)$ & $<.0001^{* *}$ \\
$\quad$ Neopterin & $2.934(1.809-4.758)$ & \\
oxLDL & $6.031(2.856-12.740)$ &
\end{tabular}

Reference category: individuals without MetS and without CAD.

(b) Multinominal regression analysis: odds ratio and 95\% CI for various inflammatory and oxidative stress markers for subjects with MetS and CAD

\begin{tabular}{lcc}
\hline & OR $(95 \% \mathrm{CI})$ & $P$-value \\
\hline Inflammatory markers & & .068 \\
$\quad$ sPLA2 & $0.670(0.435-1.030)$ & $<.0001^{* *}$ \\
Interleukin 6 & $4.716(3.120-7.130)$ & .810 \\
hsCRP & $0.967(0.738-1.268)$ & $.043^{*}$ \\
$\quad$ Fibrinogen & $0.313(0.102-0.964)$ & $.019^{*}$ \\
Oxidative stress markers & & \\
$\quad$ Myeloperoxidase & $1.842(1.105-3.072)$ & $.0001^{* *}$ \\
Neopterin & $6.209(3.549-10.864)$ & $.008^{* *}$ \\
oxLDL & $2.992(1.327-6.747)$ & \\
\hline
\end{tabular}

Reference category: individuals without MetS and without CAD.

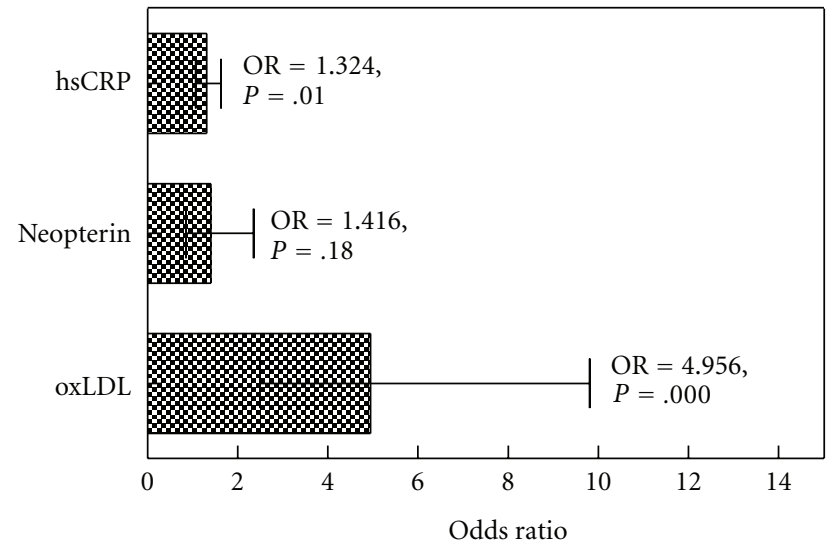

FIGURE 1: Error bar plot showing the odds ratio and 95\% CI for hsCRP, neopterin and oxLDL towards risk of developing metabolic syndrome.

As expected, mean levels of these markers were significantly different between groups 1 (without MetS) and 2 (with MetS) indicating the importance of these 3 biomarkers in identifying subjects at risk of MetS.

\section{Discussion}

Increased oxidative stress and inflammatory biomarkers are known to play an important role in the initiation and

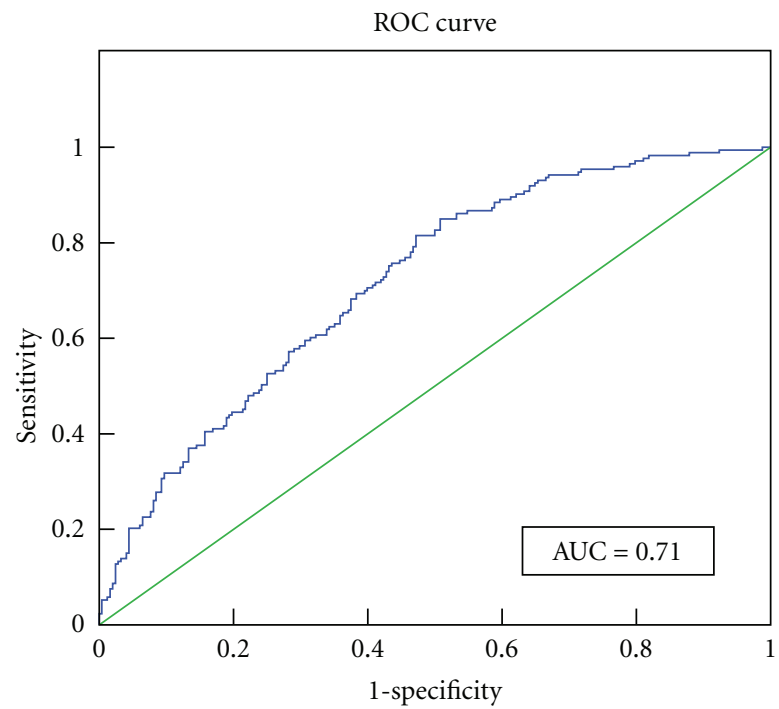

FIgURE 2: Area under the ROC curve for prediction of metabolic syndrome using a combination of three biomarkers viz oxLDL, hsCRP and neopterin

progression of atherosclerotic vascular disease [17, 18], a major cause of morbidity and mortality in the world [19]. The presence of vascular risk factors led to the identification of a unique pathophysiological condition called metabolic 
TABle 5

(a) Discriminant analysis results

\begin{tabular}{lcc}
\hline & \multicolumn{2}{c}{ Structure matrix } \\
& 1 & Contribution (\%) \\
\hline Ln_oxLDL & .746 & 37.99 \\
Ln_HsCRP & .639 & 27.87 \\
Ln_Neopterin & .522 & 18.59 \\
Ln_sPLA2 & .411 & 11.53 \\
Ln_Interleukin6 & .242 & 3.99 \\
\hline
\end{tabular}

(b) Threshold levels for the biomarkers obtained using Discriminant score

\begin{tabular}{lc}
\hline oxLDL & $39922.68 \mathrm{mU} / \mathrm{L}$ \\
hsCRP & $4.00 \mu \mathrm{g} / \mathrm{ml}$ \\
Neopterin & $7.0596 \mathrm{nmol} / \mathrm{L}$ \\
\hline
\end{tabular}

$63.4 \%$ of cases were correctly classified using discriminant analysis.

(c) Mean levels of biomarkers for discriminant groups

\begin{tabular}{llll}
\hline Variables & $\begin{array}{l}\text { Discriminant } \\
\text { group } \\
\text { (see Table 5 }(\mathrm{d}))\end{array}$ & $\begin{array}{l}\text { Mean } \pm \text { S.E } \\
\text { (retransformed mean) }\end{array}$ & $\begin{array}{l}\text { P-value for group } \\
\text { differences }\end{array}$ \\
\hline hsCRP $(\mu \mathrm{g} / \mathrm{ml})$ & 1 & $-0.066 \pm 0.080(0.9361)$ & $<.0001^{* *}$ \\
& 2 & $1.240 \pm 0.102(3.4556)$ & $<.0001^{* *}$ \\
\hline & 3 & $0.393 \pm 0.128(1.4814)$ & $<.0001^{* *}$ \\
OxLDL $(\mathrm{mU} / \mathrm{L})$ & 4 & $1.219 \pm 0.092(3.3838)$ & $<.0001^{* *}$ \\
& 1 & $10.623 \pm 0.022(41068.6362)$ & $<.0001^{* *}$ \\
& 3 & $11.005 \pm 0.028(60174.2621)$ & $<.0001^{* *}$ \\
\hline
\end{tabular}

(d) Discriminant groups

\begin{tabular}{lcc}
\hline Actual group & Predicted group & Discriminant group \\
\hline Unaffected & Unaffected & 1 \\
Unaffected & Affected & 2 \\
Affected & Unaffected & 3 \\
Affected & Affected & 4 \\
\hline
\end{tabular}

syndrome [1], now known to be characterized by elevated inflammatory markers and increased oxidative stress that can predict cardiovascular events such as the risk of myocardial infarction, stroke, and peripheral arterial disease [6, 20-22]. Importantly, the risk for Coronary Heart Disease (CHD) is markedly greater in obese individuals with MetS compared with those without it $[23,24]$. However, the association and effect of MetS on plasma biomarkers of inflammation and oxidative stress is largely unknown. In this study we tested our hypothesis that markers of inflammation and oxidative stress can differentiate between individuals with or without MetS. We also studied the individual ability of these biomarkers in predicting MetS in an otherwise healthy person.

In this first report looking at the contribution of inflammatory markers to MetS in Asian Indians from India, we find that MetS enhances inflammatory and oxidative stress and found significantly higher circulating levels of oxLDL, hsCRP, sPLA2, IL-6 and neopterin in subjects with MetS compared to those without. These findings are 
consistent with contemporary data [20-23]. To identify biomarkers with potential to diagnose MetS, we divided the study cohort into 4 groups: with/without MetS and those with/without CAD. This was done to identify novel plasma biomarkers specifically associated with MetS (Table 4(a)) and CAD (Table 4(b)). Multinomial logistic regression analysis of these groups of subjects revealed that among all the inflammatory biomarkers and markers of oxidative stress studied, hsCRP and oxLDL identified individuals with risk of developing MetS. IL-6 was the best marker of CAD risk and neopterin identified subjects with both CAD and MetS. Further analysis revealed that of the three markers only hsCRP and oxLDL identified individuals with MetS and oxLDL is the best predictor of MetS. This was despite the lack of large variations in oxLDL levels between CAD affected and unaffected groups in the study cohort.

OxLDL is known to be a potent inducer of (a) foam cells, that make a hallmark of atherosclerosis-fatty streaks, and (b) systemic inflammation alongside propagation of atherosclerosis $[12,14,17,25]$. This may explain our robust findings with respect to this biomarker.

ROC curve analysis using predictive probability models revealed that a combination of oxLDL, hsCRP and neopterin identifies $71 \%$ of individuals with MetS. Discriminant analysis revealed the percent contribution of each of the biomarkers studied towards MetS. OxLDL contributed maximally and on the basis of the discriminant scores $63.4 \%$ of the cohort was classified accurately (Table 5(a)). Having obtained threshold values of ox-LDL for recognition of MetS using discriminant score, we found that subjects with higher values showed a 5-fold greater risk of developing MetS. The propensity of the LDL particles for oxidation decides the concentration of oxidized LDL in circulation. Small dense LDL contains fewer anti-oxidants, and therefore is more prone to oxidation [14]. MetS has been reported to be associated with a higher prevalence of small dense LDL [26] and among ethnic groups, Asian Indians are reported to show higher levels of small dense LDL particles [27, 28]. As found in our research, the fact that people with MetS are at an increased risk of macro vascular disease and death [29] appears to further explain the strong association of MetS with high concentrations of oxLDL. Our findings are concurrent with studies in various populations [14, 3032], but this is the first report on Asian Indians from India.

In conclusion, our population data from families of Asian Indians with strong family histories of CAD shows that metabolic syndrome is associated with high inflammatory and oxidative stress. Diagnosis of MetS adds weight to the comprehensive CAD risk assessment of individuals. Further, novel biomarkers oxLDL, hsCRP and neopterin can be utilized to identify people with MetS in the Asian Indian population. OxLDL has been identified as the best predictive biomarker for MetS in Asian Indians and contributes maximally to the dysmetabolic state in comparison to all other biomarkers. Further research will validate its use to diagnose people with MetS compared to internationally defined criteria to ultimately yield a cost-effective method for MetS diagnosis and CAD risk stratification.

\section{Acknowledgments}

The authors gratefully acknowledge the Tata Social Welfare Trust, Elizabeth and Emmanuel Kaye Foundation, and the trustees of TRI India \& London for supporting the IARS. The authors thank all the investigators, staff and participants of the IARS from Narayana Hrudayalaya, Bangalore, and Asian heart center, Mumbai, for their contribution and the members of the administrative teams for their assistance. The authors are grateful to the patients, their family members, and the unaffected subjects for participating in the study. The authors thank Dr. Usha Narayan for editorial assistance. The authors have no conflict of interest to disclose.

\section{References}

[1] G. M. Reaven, "Banting Lecture 1988. Role of insulin resistance in human disease. 1988," Nutrition, vol. 13, no. 1, pp. 65-66, 1997.

[2] K. G Alberti and P. Z. Zimmet, "Definition, diagnosis and classification of diabetes mellitus and its complications-part 1: diagnosis and classification of diabetes mellitus. Provisional report of a WHO consultation," Diabetic Medicine, vol. 15, no. 7, pp. 539-553, 1998.

[3] "Third Report of the National Cholesterol Education Program (NCEP) Expert Panel on detection, evaluation, and treatment of high blood cholesterol in adults (Adult Treatment Panel III)," Circulation, vol. 106, no. 25, pp. 3143-3421, 2002.

[4] K. G. Alberti, P. Zimmet, and J. Shaw, "The metabolic syndrome-a new worldwide definition,” Lancet, vol. 366, no. 9491, pp. 1059-1062, 2005.

[5] R. H. Eckel, S. M. Grundy, and P. Z. Zimmet, "The metabolic syndrome," Lancet, vol. 365, no. 9468, pp. 1415-1428, 2005.

[6] A. Festa, R. D’Agostino Jr., G. Howard, L. Mykkänen, R. P. Tracy, and S. M. Haffner, "Chronic subclinical inflammation as part of the insulin resistance syndrome: the insulin resistance atherosclerosis study (IRAS)," Circulation, vol. 102, no. 1, pp. 42-47, 2000.

[7] P. M. Ridker, P. W. F. Wilson, and S. M. Grundy, "Should C-reactive protein be added to metabolic syndrome and to assessment of global cardiovascular risk?" Circulation, vol. 109, no. 23, pp. 2818-2825, 2004.

[8] S. Fujioka, Y. Matsuzawa, K. Tokunaga, and S. Tarui, "Contribution of intra-abdominal fat accumulation to the impairment of glucose and lipid metabolism in human obesity," Metabolism, vol. 36, no. 1, pp. 54-59, 1987.

[9] A. G. Pittas, N. A. Joseph, and A. S. Greenberg, "Adipocytokines and insulin resistance," Journal of Clinical Endocrinology and Metabolism, vol. 89, no. 2, pp. 447-452, 2004.

[10] M. K. Rutter, J. B. Meigs, L. M. Sullivan, R. B. D’Agostino, and P. W. F. Wilson, "C-reactive protein, the metabolic syndrome, and prediction of cardiovascular events in the Framingham offspring study," Circulation, vol. 110, no. 4, pp. 380-385, 2004.

[11] Y. Matsuzawa, T. Funahashi, S. Kihara, and I. Shimomura, "Adiponectin and metabolic syndrome," Arteriosclerosis, Thrombosis, and Vascular Biology, vol. 24, pp. 29-33, 2004.

[12] R. Masella, R. Varì, M. D’Archivio et al., “Oxidised LDL modulate adipogenesis in 3T3-L1 preadipocytes by affecting the balance between cell proliferation and differentiation," FEBS Letters, vol. 580, no. 10, pp. 2421-2429, 2006. 
[13] B. A. Maddux, W. See, J. C. Lawrence Jr., A. L. Goldfine, I. D. Goldfine, and J. L. Evans, "Protection against oxidative stressinduced insulin resistance in rat 16 muscle cells by micromolar concentrations of $\alpha$-lipoic acid," Diabetes, vol. 50, no. 2, pp. 404-410, 2001.

[14] P. Holvoet, D. H. Lee, M. Steffes, M. Gross, and D. R. Jacobs, "Association between circulating oxidized low-density lipoprotein and incidence of the metabolic syndrome," Journal of the American Medical Association, vol. 299, no. 19, pp. 22872293, 2008.

[15] G. P. Van Guilder, G. L. Hoetzer, J. J. Greiner, B. L. Stauffer, and C. A. DeSouza, "Influence of metabolic syndrome on biomarkers of oxidative stress and inflammation in obese adults," Obesity, vol. 14, no. 12, pp. 2127-2131, 2006.

[16] A. Misra and N. K. Vikram, "Insulin resistance syndrome (metabolic syndrome) and Asian Indians," Current Science, vol. 83, no. 12, pp. 1483-1496, 2002.

[17] S. I. Toshima, A. Hasegawa, M. Kurabayashi et al., "Circulating oxidized low density lipoprotein levels: a biochemical risk marker for coronary heart disease," Arteriosclerosis, Thrombosis, and Vascular Biology, vol. 20, no. 10, pp. 2243-2247, 2000.

[18] P. Libby, P. M. Ridker, and A. Maseri, "Inflammation and atherosclerosis," Circulation, vol. 105, no. 9, pp. 1135-1143, 2002.

[19] J. L. Murray and A. D. Lopez, The Global Burden of Disease: a Comprehensive Assessment of Mortality and Disability from Diseases, Injuries, and Risk Factors in 1990 and Projected to 2020, The Harvard School of Public Health, Boston, Mass, USA, 1996.

[20] P. M. Ridker, N. Rifai, L. Rose, J. E. Buring, and N. R. Cook, "Comparison of C-reactive protein and low-density lipoprotein cholesterol levels in the prediction of first cardiovascular events," New England Journal of Medicine, vol. 347, no. 20, pp. 1557-1565, 2002.

[21] B. Hansel, P. Giral, E. Nobecourt et al., "Metabolic syndrome is associated with elevated oxidative stress and dysfunctional dense high-density lipoprotein particles displaying impaired antioxidative activity," Journal of Clinical Endocrinology and Metabolism, vol. 89, no. 10, pp. 4963-4971, 2004.

[22] M. Jacobs, M. M. J. Van Greevenbroek, C. J. H. Van Der Kallen et al., "Low-grade inflammation can partly explain the association between the metabolic syndrome and either coronary artery disease or severity of peripheral arterial disease: the CODAM study," European Journal of Clinical Investigation, vol. 39, no. 6, pp. 437-444, 2009.

[23] J.-P. Després, "Inflammation and cardiovascular disease: is abdominal obesity the missing link?" International Journal of Obesity, vol. 27, supplement 3, pp. S22-S24, 2003.

[24] H. M. Lakka, D. E. Laaksonen, T. A. Lakka et al., "The metabolic syndrome and total and cardiovascular disease mortality in middle-aged men," Journal of the American Medical Association, vol. 288, no. 21, pp. 2709-2716, 2002.

[25] P. Holvoet, "Oxidized LDL and coronary heart disease," Acta Cardiologica, vol. 59, no. 5, pp. 479-484, 2004.

[26] B. Lamarche, "Abdominal obesity and its metabolic complications: implications for the risk of ischaemic heart disease," Coronary Artery Disease, vol. 9, no. 8, pp. 473-481, 1998.

[27] E. A. Enas and A. Senthilkumar, "Coronary artery disease in Asian Indians: an update and review," International Journal of Cardiology, vol. 1, p. 2, 2001.

[28] Mission statement. American Association of Physicians of Indian Origin Coronary Artery Disease Committee, 2002, http://www.aapio.org/.
[29] M. Trevisan, J. Liu, F. B. Bahsas, and A. Menotti, "Syndrome X and mortality: a population-based study," American Journal of Epidemiology, vol. 148, no. 10, pp. 958-966, 1998.

[30] P. Holvoet, S. B. Kritchevsky, R. P. Tracy et al., "The metabolic syndrome, circulating oxidized LDL, and risk of myocardial infarction in well-functioning elderly people in the health, aging, and body composition cohort," Diabetes, vol. 53, no. 4, pp. 1068-1073, 2004.

[31] V. Sigurdardottir, B. Fagerberg, and J. Hulthe, "Circulating oxidized low-density lipoprotein (LDL) is associated with risk factors of the metabolic syndrome and LDL size in clinically healthy 58-year-old men (AIR study)," Journal of Internal Medicine, vol. 252, no. 5, pp. 440-447, 2002.

[32] S. I. Yamagishi, H. Matsuoka, S. Kitano et al., "Elevated circulating oxidized LDL levels in Japanese subjects with the metabolic syndrome," International Journal of Cardiology, vol. 118, no. 2, pp. 270-272, 2007. 


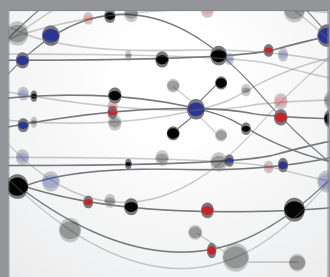

The Scientific World Journal
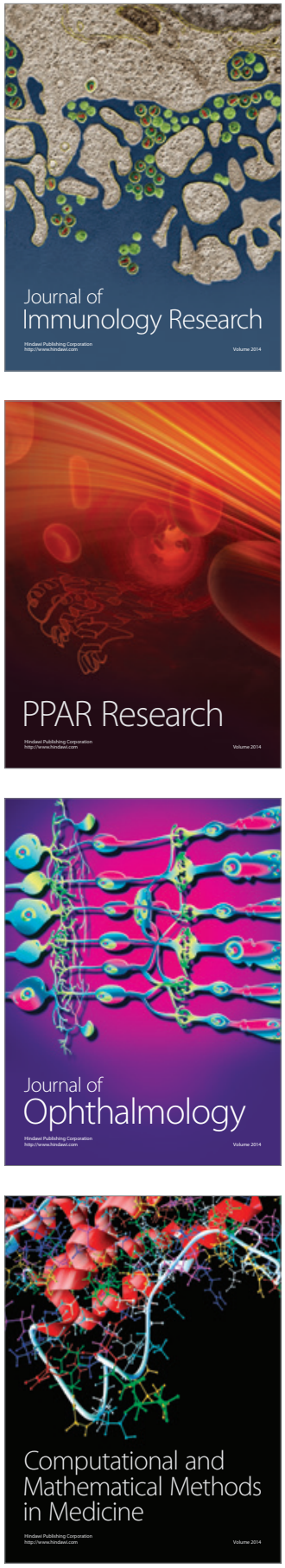

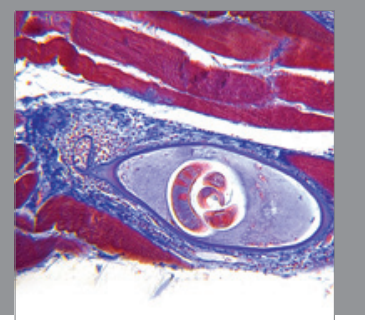

Gastroenterology

Research and Practice
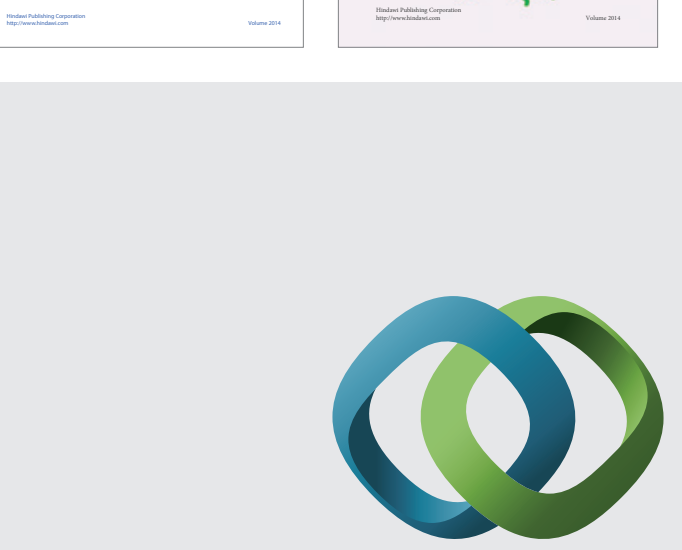

\section{Hindawi}

Submit your manuscripts at

http://www.hindawi.com
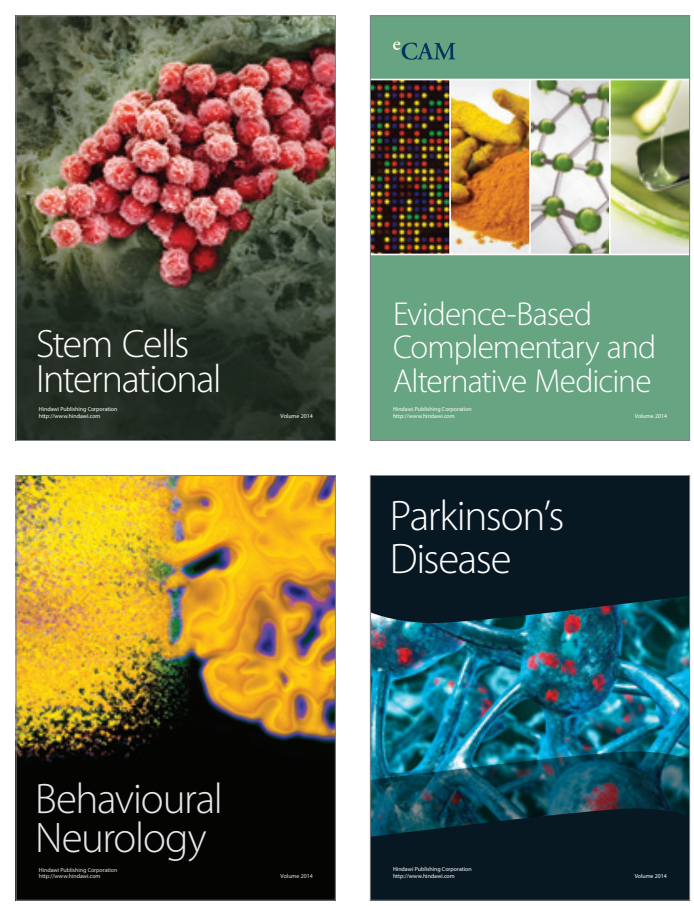

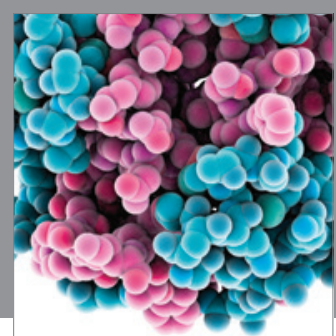

Journal of
Diabetes Research

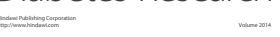

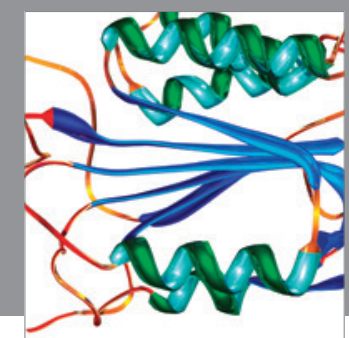

Disease Markers
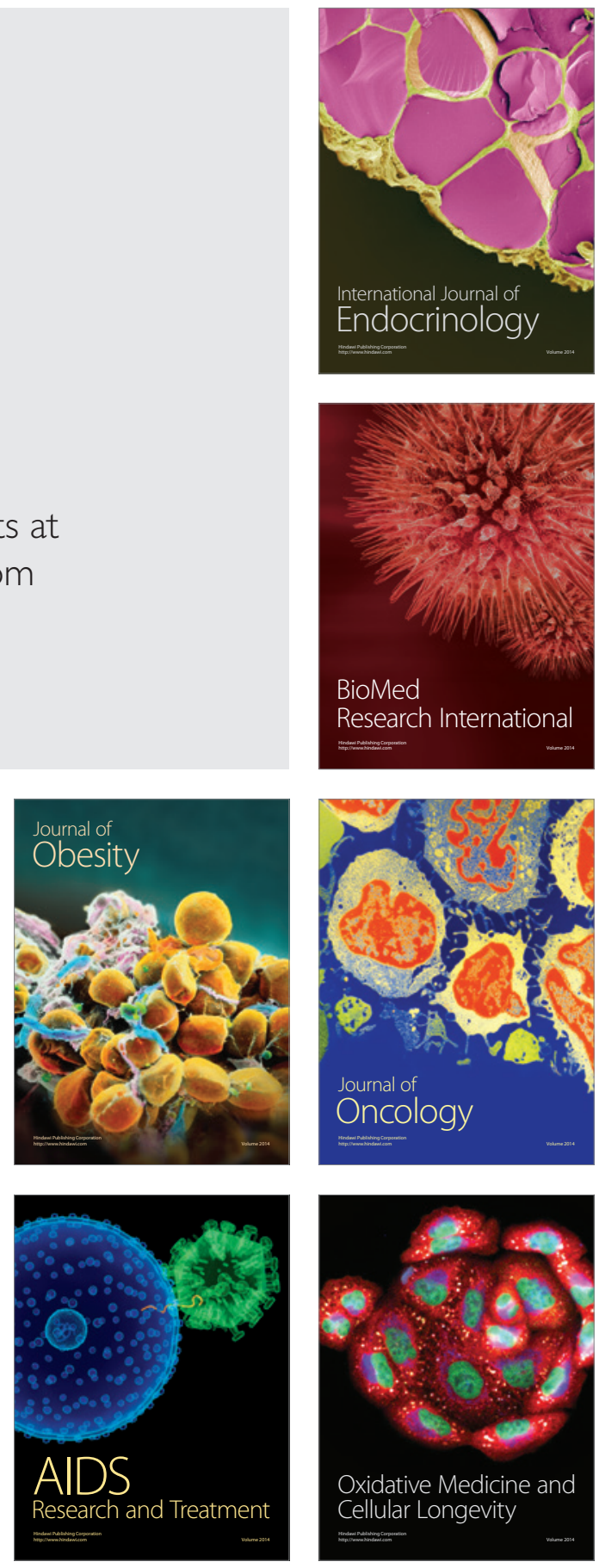